\title{
Polarization-Independent Dilated InP-Based Space Switch with Low Crosstalk
}

\author{
D. H. P. Maat, Y. C. Zhu, F. H. Groen, H. van Brug, H. J. Frankena, and X. J. M. Leijtens
}

\begin{abstract}
A polarization-independent dilated switch with a low crosstalk level has been realized. In this switch, the crosstalk signal is attenuated by using controllable electrooptical attenuators which do not disturb the input signal. A reverse bias of $20 \mathrm{~V}$ on the attenuators reduces the crosstalk value of the dilated switch from $-32 \mathrm{~dB}$ to $-40 \mathrm{~dB}$. The excess loss of the total switch is about $9 \mathrm{~dB}$.
\end{abstract}

Index Terms-Electrooptic switches, integrated optics, photonic integrated circuits, semiconductor waveguides, wavelength-division multiplexing.

\section{INTRODUCTION}

$\mathbf{O}$ PTICAL space switches play a key role in advanced optical networks, both for wavelength-division-multiplexed (WDM) and single-wavelength applications. The demands WDM systems impose on their components are high: crosstalk levels better than $-35 \mathrm{~dB}$ are required to obtain a proper system functionality, even for a small number of (crosstalk-introducing) components [1]. The best crosstalk levels of multiwavelength space switches on InP reported so far are about $-20 \mathrm{~dB}$ for both polarization states [2], [3]. This level can be reduced by placing the switch in a dilated scheme, which theoretically lowers the crosstalk level to the square of the single-switch crosstalk $(-40 \mathrm{~dB})$. In practice, the crosstalk is only reduced to a level of $-30 \mathrm{~dB}$ [4], [5], which is still too high according to Goldstein [1].

To satisfy the high demands described above, we reduced the crosstalk of the dilated switch further by using electroabsorbing phase-shifting sections which were placed in the connecting waveguides between the two switch sections in the dilated switch as controllable absorbers. By operating only the absorbers in the crosstalk-path, a significant reduction of the crosstalk is achieved.

\section{DESIGN AND FABRICATION}

The design of a single Mach-Zehnder interferometer (MZI)-based $2 \times 2$ switch, which is used as a building block of the dilated switch, is shown in Fig. 1. In this switch, multimode interference couplers (MMI's) $(16 \times 400 \mu \mathrm{m})$ are used as $3-\mathrm{dB}$ couplers, in between which the phase-shifting sections are

Manuscript received March 29, 1999; revised November 15, 1999. This was supported by IOP Electro-Optics IEO94003 of the Dutch Government and by the E.C. ACTS project AC332 "APEX."

D. H. P. Maat, Y. C. Zhu, F. H. Groen, H. van Brug, and H. J. Frankena are with the Optics Research Group, Department of Applied Physics, Delft University of Technology, NL-2600 GA Delft, The Netherlands.

X. J. M. Leijtens is with the Department of Information Technology and Systems, Delft University of Technology, NL-2600 GA Delft, The Netherlands.

Publisher Item Identifier S 1041-1135(00)01961-3.

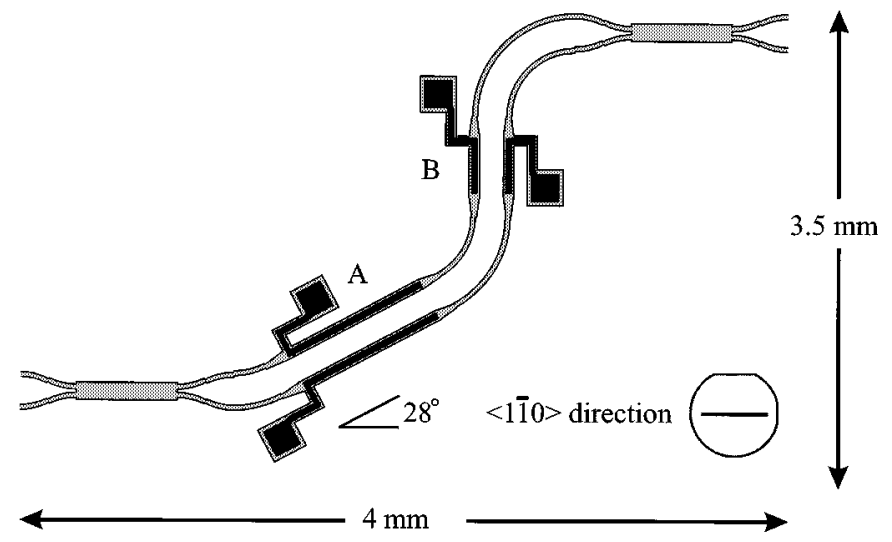

Fig. 1. Layout of the single $2 \times 2$ MZI-switch.

positioned. Each phase-shifting section consists of two parts. With part A $(2 \mathrm{~mm})$, placed at an angle of $28^{\circ}$ with respect to the $[1 \overline{1} 0]$ direction, roughly polarization-independent switching is obtained while part B $(0.5 \mathrm{~mm})$, placed in the $[110]$ direction, serves to correct for small deviations originating in variations in doping levels and layer thickness [6].

As controllable absorbers, single phase-shifting sections are used by employing the electroabsorption effect. Since they will only operate in the crosstalk path, the phase and amplitude of the signal applied to the switch will not be affected. Fig. 3 shows the combination of the $2 \times 2$ switches and the attenuating elements (with a length of $0.5 \mathrm{~mm}$ ) used in the dilated switch.

All waveguides in the dilated switch have a width of $3 \mu \mathrm{m}$ to provide low loss, except for the phase-shifting sections which have a width of $4.5 \mu \mathrm{m}$ to enable the alignment of the $2-\mu$ m-wide contacts. All curved waveguides in the switch have a radius of $500 \mu \mathrm{m}$. The small wavelength dependence of the electrooptical effects and the MMI's in the 1550-nm telecommunication window ensures wavelength-insensitive operation of the switch in this window.

The growth of the InGaAsP-InP-InGaAs layer stack is performed on a (001) oriented $\mathrm{n}^{+}$substrate. The thickness and doping level of the various layers are shown in Fig. 2. The InGaAsP light-guiding layer is n-doped such that the switch is already close to polarization-independent operation and at the same time has low loss.

The waveguides of the dilated switch are realized with a $\mathrm{CH}_{4}-\mathrm{H}_{2} / \mathrm{O}_{2} \mathrm{RIE} /$ descum process, using (PECVD) silicon-nitride masking. To have electrical insulation between the different phase-shifting sections, the waveguides at the front and at the end of each section have been etched down to the pn-junction over a length of $250 \mu \mathrm{m}$. The pn-junction is passivated by cured polyimide. The fabrication of the switch 


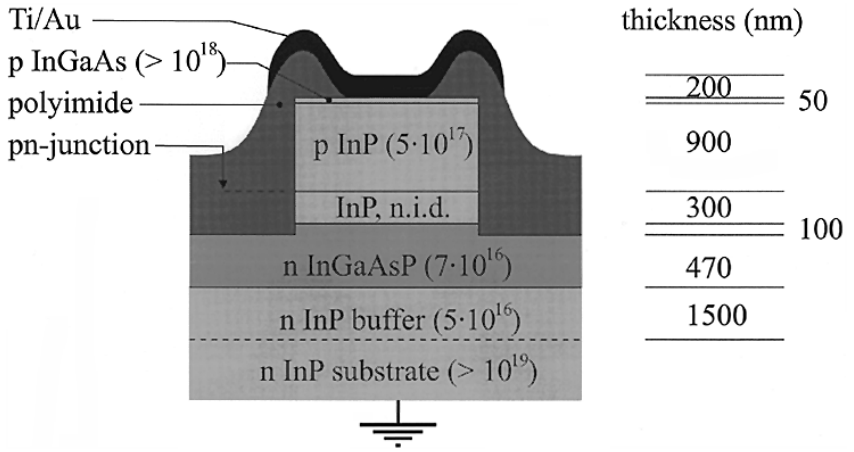

Fig. 2. A cross section of a phase-shifting section (doping levels in $\mathrm{cm}^{-3}$ ).

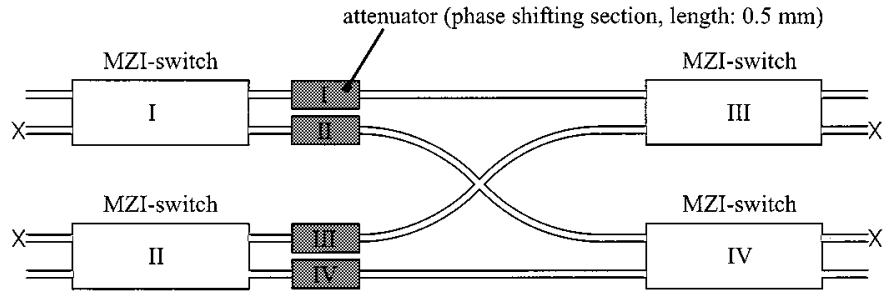

Fig. 3. Schematic layout of the dilated switch consisting of four single $2 \times 2$ switches and four attenuators in between the switches. The dimensions of the dilated switch are: $12 \times 4 \mathrm{~mm}$. The inputs and outputs marked with a cross are idle.

is finished by depositing Ti-Au contacts on the phase-shifting sections using a lift-off technique.

\section{MeAsuREMEnT Results}

The measurements are performed at a wavelength of 1.5 $\mu \mathrm{m}$. A microscope objective was used to couple light into the waveguides. At the output side a tapered single-mode fiber was used. In Table I, the crosstalk levels of the different switches in the dilated switch are given. The attenuation of the absorbers as a function of the applied reverse bias is given in Fig. 4, which shows that attenuation starts at a reverse bias of $5 \mathrm{~V}$.

The switching curves of the dilated switch are given in Fig. 5 . When no bias is applied to the attenuators $(0 \mathrm{~V})$, the crosstalk of the dilated switch is $-34.8 \mathrm{~dB}$ for TE and -31.4 for TM polarization. The on-chip loss of the dilated switch for both TE and TM polarization is $9 \mathrm{~dB}$ which is large with respect to previously reported dilated switches without absorbers (on-chip loss: $5.5 \mathrm{~dB}$ ) [4]. The extra losses introduced by the attenuating elements (at 0 V), caused by their electrical insulation regions, are about $1 \mathrm{~dB}$ for TM polarization and can be neglected for TE polarization. The additional losses are ascribed to variations in the dimensions of the MMI's and to layer thickness variations. These are expected to be removed in a next fabrication run.

The crosstalk of the dilated switch is reduced by applying a reverse bias to the proper attenuators: in the bar-state, attenuators II and III are used, while for the cross state attenuators I and IV have to be employed. In these cases, the crosstalk signal is absorbed while the signal applied to both inputs of the dilated switch is not affected.

In Fig. 5, both the bar-port switching curves, using attenuators I and IV, and the cross-port curves using attenuators II and III are given for different reverse bias values applied to the attenuator.

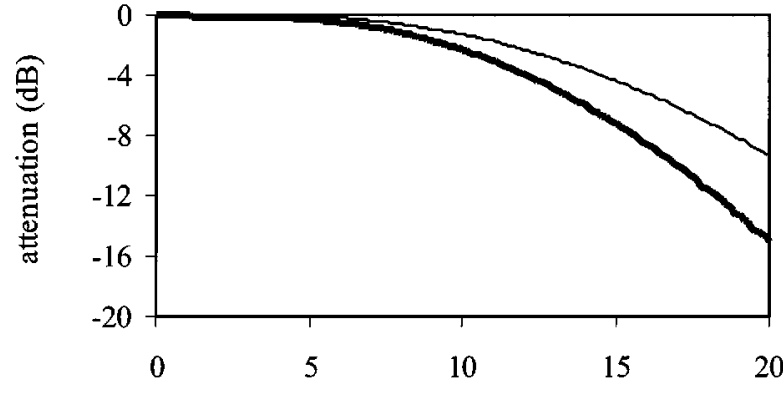

reverse bias $(\mathrm{V})$

Fig. 4. The attenuation of a single phase-shifting section (length: $0.5 \mathrm{~mm}$ ) as a function of the applied reverse bias (thin line: TE polarization; bold line: TM polarization).

TABLE I

POLARIZATION-INDEPENDENT CROSSTALK

LEVELS IN Decibels of tHE Switches IN THE Dilated Switch (NUMBERING AS IN FIG. 3)

\begin{tabular}{|c|c|c|c|c|}
\cline { 2 - 5 } \multicolumn{1}{c|}{} & Switch I & Switch II & Switch III & Switch IV \\
\hline TE & -20.3 & -21.4 & -20.1 & -20.0 \\
\hline TM & -21.4 & -17.6 & -18.1 & -19.9 \\
\hline
\end{tabular}
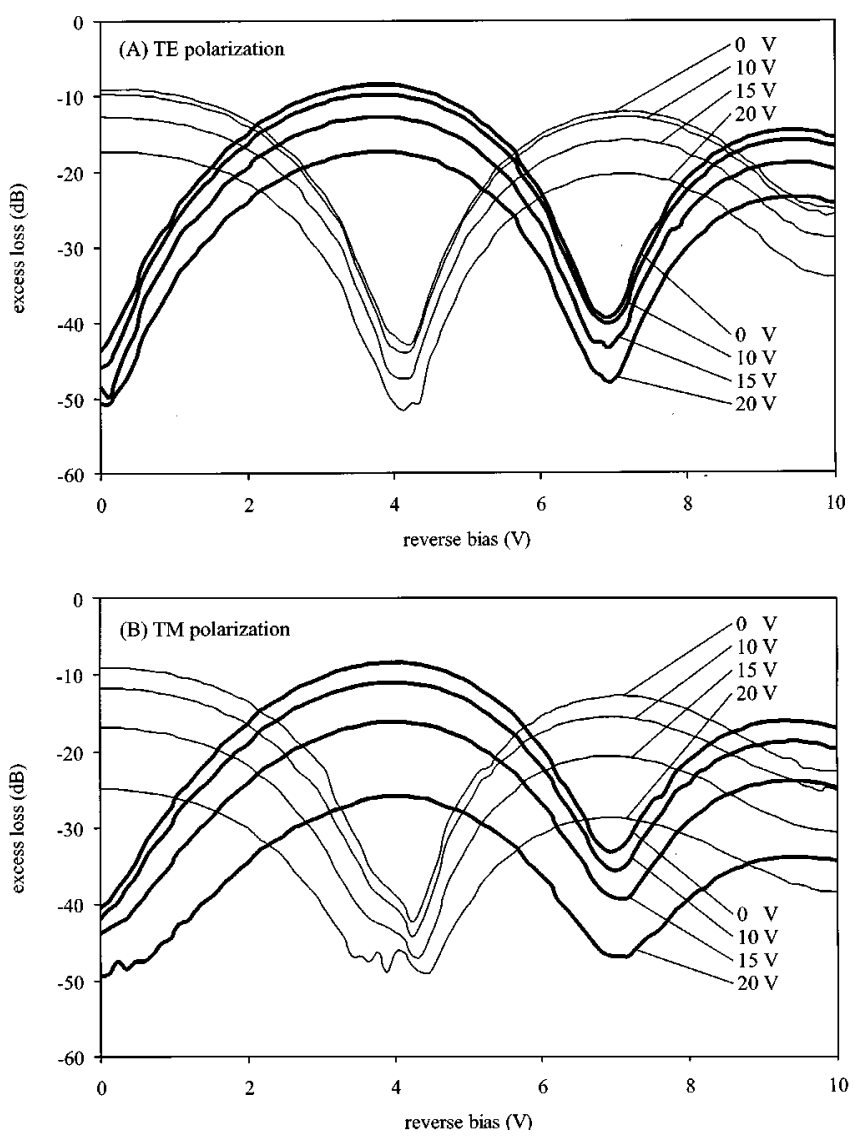

Fig. 5. Switching curves of the dilated switch for different reverse bias values applied to the attenuators. Bold lines: bar port, output utilizing attenuators I and IV, thin lines: cross port, output utilizing attenuators II and III. (A) TE polarization. (B) TM polarization.

The best crosstalk level in the bar-state, achieved when a reverse bias of $20 \mathrm{~V}$ is applied to attenuators II and III, is given in Fig. 5 by the difference between the top bar-port curve $(0 \mathrm{~V}$ on attenuators I and IV: no disturbance of input-signal) and the 
TABLE II

Crosstalk Levels in Decibels of the Dilated Switch With and WITHOUT USING THE ATTENUATOR

\begin{tabular}{|c|c|c|}
\cline { 2 - 3 } \multicolumn{1}{c|}{} & without attenuator & attenuator $(20 \mathrm{~V})$ \\
\hline TE & -34.8 & -41.6 \\
\hline TM & -31.4 & -40.1 \\
\hline
\end{tabular}

lowest cross-port curve (-20 V on attenuators II and III: maximal crosstalk absorption). Using the same method for the cross state, a crosstalk reduction of $6.8 \mathrm{~dB}$ for TE polarization and 8.7 $\mathrm{dB}$ for TM polarization is found when applying a reverse bias of $20 \mathrm{~V}$ to the attenuators. An overview of all crosstalk values is given in Table II.

As can be seen in both Figs. 4 and 5, TM attenuation is larger than TE attenuation; in this way, TM crosstalk, which is usually worse than TE crosstalk, gets closer to the TE level when using the absorbers.

\section{CONCLUSION}

A polarization-independent dilated switch containing electrooptical controllable attenuators in between the switches has been fabricated and measured. The dilated switch has a crosstalk level of $-31.4 \mathrm{~dB}$. When using the attenuators, the crosstalk is reduced by $8.7 \mathrm{~dB}$, resulting in a polarization-independent crosstalk of $-40 \mathrm{~dB}$.

\section{REFERENCES}

[1] E. L. Goldstein, L. Eskildsen, and A. F. Elrefaie, "Scaling limitations in transparent optical networks due to low-level crosstalk," IEEE Photon. Technol. Lett., vol. 7, pp. 93-94, Jan. 1995.

[2] R. Krähenbühl, R. Kyburz, W. Vogt, M. Bachmann, T. Brenner, E. Gini, and H. Melchior, "Low-loss polarization-insensitive InP-InGaAsP optical space switches for fiber optical communication'," IEEE Photon. Technol. Lett., vol. 8, no. 5, pp. 632-634, 1996.

[3] T. Uitterdijk, H. van Brug, F. H. Groen, H. J. Frankena, C. G. M. Vreeburg, and J. J. G. M. van der Tol, "'Integrable polarization insensitive InGaAsP Mach-Zender switch'," in Integrated Photonic Research: OSA Technical Digest Series, 1996, vol. 6, pp. 486-489.

[4] T. Uitterdijk, C. G. M. Vreeburg, D. H. P. Maat, F. H. Groen, H. van Brug, and I. Moerman, "Dilated, polarization insensitive InP based space switch'," in Proc. 8th Eur. Conf. Integrated Optics (ECIO'97), Stockholm, Sweden, 1997, pp. 551-554.

[5] R. Krähenbühl, W. Vogt, W. Hunziker, H. Schneibel, R. Bauknecht, E. Gini, and H. Melchior, "Low loss polarization insensitive high speed InP-InGaAsP optical space switch modules fully packaged with electronic driver and single mode fibers," in Proc. 8th Eur. Conf. Integrated Optics (ECIO'97), Stockholm, Sweden, 1997, pp. 466-469.

[6] D. H. P. Maat, C. G. P. Herben, M. K. Smit, F. H. Groen, H. van Brug, H. J. Frankena, and I. Moerman, "Polarization independent InP-based switch with relaxed fabrication tolerances'," in Integrated Photonics Research: OSA Technical Digest Series, 1998, vol. 4, pp. 246-248. 